\title{
Self-relevance effects and label choice: Strong variations in label-matching performance due to non-self-relevant factors
}

\author{
Gregory L. Wade ${ }^{1} \cdot$ Timothy J. Vickery ${ }^{1}$
}

Published online: 20 March 2017

(C) The Psychonomic Society, Inc. 2017

\begin{abstract}
Merely associating one's self with a stimulus may be enough to enhance performance in a label-matching paradigm (Sui, He, \& Humphreys, 2012), implying prioritized processing of self-relevant stimuli. For instance, labeling a square as SELF and a circle as OTHER yields speeded performance when verifying square-SELF compared with circle-OTHER label matches. The precise causes of such effects are unclear. We propose that prioritized processing of label-matches can occur for reasons other than self-relevance. Here, we employ the label-matching paradigm to show similar benefits for nonself-relevant labels (SNAKE, FROG, and GREG) over a frequently employed, non-self-relevant control label (OTHER). These benefits suggest the possibility that self-relevance effects in the label-matching paradigm may be confounded with other properties of labels that lead to relative performance benefits, such as concreteness. The size of selfrelevance effects may be overestimated in prior work employing the label-matching paradigm, which calls for greater care in the choice of control labels to determine the true magnitude of self-relevance effects. Our results additionally indicate the possibility of a powerful effect of concreteness (and related properties) on associative memory performance.
\end{abstract}

Keywords Associative learning · Self-relevance · Self-identification $\cdot$ Concreteness $\cdot$ Label matching

Gregory L. Wade

gwade@psych.udel.edu

1 University of Delaware, 108 Wolf Hall, Newark, DE 19716, USA
We often encounter situations in which we associate our sense of self with an arbitrary stimulus. For instance, names are abstract self-representations, games often require assigning arbitrary tokens to serve as one's in-game self-representation, and team sports often require associating oneself with a novel group identity. Does such an assignment of identity alter cognition surrounding that stimulus's representation? Evidence suggests that it does, resulting in improved processing and performance related to those stimuli, a finding labeled the 'self-relevance effect' (Sui, Liu, Wang, \& Han, 2009; Sui, He, \& Humphreys, 2012). However, the extent to which self-representation, per se, is privileged by cognition is unclear, because the existing literature on this topic has primarily involved comparing strongly self-relevant labels with a limited set of weakly self-relevant labels. Thus, it is possible that the "self-relevance effect" may, instead, reflect access to generic mechanisms that are not exclusive to self-identified stimuli. Here, we examine the possibility that the assignment of self-relevance may not be entirely responsible for the observed benefits over non-self-relevant stimuli. We do this by comparing self-labels against a novel set of labels that are not strictly self-relevant, but share conceptual features with self-labels.

Self-association of a stimulus appears to have a variety of effects on performance. For instance, humans show an improved recognition of their own face over other familiar faces (Keenan, Wheeler, Gallup, \& Pascual-Leone, 2000; Kircher et al., 2000; Turk et al., 2002), and preferentially store verbal attributes and traits if they associate the information to themselves (Kelley et al., 2002; Johnson et al., 2002). Self-association also has well-known benefits to memory for self-associated compared with non-self-associated stimuli (e.g., Symons \& Johnson, 1997; Macrae, Moran, Heatherton, Banfield, \& Kelley, 2004).

Sui et al. (2012), and subsequent studies, recently demonstrated a striking phenomenon arising from minimal training that assigned personal relevance to arbitrary visual stimuli 
(geometric shapes). They found enhanced processing of stimuli arbitrarily designated as self-relevant using a label-matching paradigm. In their experiments, one shape was randomly assigned to represent the participant, labeled: YOU, while other shapes were assigned non-self-labels, such as FRIEND, MOTHER, STRANGER, NONE, (Sui et al., 2012) or UNFAMILIAR (Sui, Liu, Mevorach, \& Humphreys, 2015). After the labels were trained in a very brief associative learning task, subjects were shown pairs consisting of labels and shapes and asked to respond whether they 'matched' or 'mismatched.' Subjects confirmed label matches of the self-relevant stimulus faster, and with higher accuracy, compared with label matches of other classes, despite equalized training experience with each of the label-shape pairs.

Based on these and supporting findings (Sui \& Humphreys, 2015a; Sui \& Humphreys, 2015b), Humphreys and Sui (2016) have hypothesized the existence of a "SelfAttention Network," and suggest that activation of this network enhances a variety of cognitive functions. They proposed that this network acts to prioritize self-relevant information by means of enhancing the saliency of the associated stimuli at multiple levels, even if self-relevance is arbitrarily defined. According to their account, self-relevance effects on salience modulate a broad range of cognitive processes spanning perception, memory and decision-making.

The performance differences exhibited to self-relevant compared with non-self-relevant labels in prior work are very clear; however, an important and, in our view, unresolved issue remains: is self-relevance the exclusive reason for observed performance benefits? At least two alternatives are possible. First, self-relevance may be one of multiple means for semantic properties of associates to engage prioritization mechanisms. Second, properties of labels that define self-relevance may be confounded with other factors, and one of those other (more generic) factors, rather than self-relevance itself, may be the underlying reason for some or all of the observed effects.

The label-matching findings of Sui and colleagues are compelling, because they involve abstract associations between concepts and stimuli, learned over short exposures. Extreme exposure, overlearned associations, or even innate bottom-up mechanisms may drive other putative demonstrations of semantic factors on performance, such as own-name effects (Tacikowski et al., 2011), effects of attractiveness (O’Doherty, et al., 2003; Sui \& Liu, 2009), or the prioritization of threatening stimuli (Notebaert, Crombez, Van Damme, De Houwer, \& Theeuwes, 2011). The label-matching paradigm offers the possibility of access to a powerful top-down semantic influence on performance with minimal introduction of such confounding factors, and thus observations of selfrelevant effects in this context provide some of the most compelling evidence of broad performance benefits exclusive to self-relevant stimuli, to date. Despite these advantages, it is difficult to avoid the fact that the labels, themselves, are subject to many difficult to control factors related to experience and semantic properties. Further, the exact meaning of 'self-relevant' is unclear.

Most work with the label-matching paradigm and similar methods have shown 'self-relevance' effects using either selfidentification (the self is directly linked to the label), or social affiliation (either in-group label or biological relationship). With respect to the possibility that self-relevance may be one of a broader class of semantic properties that induce performance benefits to associated stimuli, we begin in this paper by exploring the possibility that a different semantic property, threat, may do the same. Threatening stimuli, like self-identified and socially affiliated stimuli, are also known to produce variations in cognitive performance (Cisler, Bacon, \& Williams, 2009). Threat-related stimuli have been shown to automatically capture attention in rapid serial visual presentation, (Most, Chun, Widders, \& Zald, 2005; Smith, Most, Newsome, \& Zald, 2006). Threatening stimuli are also identified more quickly in visual search compared with threat-irrelevant stimuli (Ohman, Flykt, \& Esteves, 2001). To our knowledge, no one has examined whether arbitrarily associating threatening concepts with a stimulus can elicit similar effects as associating self-concepts with a stimulus. Thus, we explore this possibility in our first experiment. It is worth noting that threat can be construed as a variety of self-relevance, in that some aspect of the self is what is threatened. Examining whether threat can elicit similar responses in label-matching will either expand or constrain the limits of 'self-relevance' that can access conceptual prioritization mechanisms.

The second major possibility, that self-relevance is confounded with other properties that may elicit similar benefits to performance, can be motivated by considering effects of concreteness and imageability on performance. Word imageability refers to the ease of forming a mental image based on a word, while concreteness is a related concept that refers to the extent to which the referent of a word can be physically instantiated (Paivio, Yuille, \& Madigan, 1968). While imageability and concreteness are highly correlated, they are not the same-emotional words like "sad" are imageable but not concrete, for instance. ${ }^{1}$ Importantly, these factors influence performance involving word stimuli. Highly imageable and/or concrete words are associated with better memory recall (e.g., Paivio, 1986; Tse \& Altarriba, 2007; Paivio, Yuille, \& Smythe, 1966), speed of word naming (e.g., de Groot, 1989), and speed and accuracy of lexical decision tasks (e.g., Bleasdale, 1987; de Groot, 1989; Whaley, 1978; Lagishetti \& Goswami, 2012). Imaging research has also uncovered evidence that there are distinct neural responses associated with concrete and

\footnotetext{
"We generally use "concreteness" and "imageability" interchangeably in this paper, as they are highly correlated, have similar effects on word processing, and the distinction is not relevant for our label choices.
} 
abstract words (Binder, Westbury, McKiernan, Possing, \& Medler, 2005; Kiehl et al., 1999). In the label matching paradigm, many of the concepts evoked by control words used in prior work relating label-matching performance to selfrelevance are arguably more abstract or less specific (OTHER, UNFAMILIAR, STRANGER, FRIEND) than the notion of the self, especially as evoked in these paradigms (e.g., "the circle represents your self" is more specific and concrete than the word "self" in isolation). Here, we raise the possibility that much of the self-benefit previously demonstrated may be due to confounds with concreteness, imageability, and/or specificity of the SELF label.

To examine these factors, we conducted a series of experiments using the label matching paradigm. First, we replicated the basic self-relevance effect in a label-matching paradigm (using the labels SELF/FRIEND/OTHER, Experiment 1A). Next, we examined whether a different kind of highly salient semantic characteristic, threat, can produce similar effects (using SELF/STRANGER/OTHER and SELF/SNAKE/ OTHER, Experiments 1B and 1C, respectively). We observed that the label SNAKE produces a similar effect on performance as SELF compared with OTHER, but that a more abstract and more difficult to image, but still threatening label (a "dangerous" STRANGER) does not. To examine the possibility that the oddball status of SNAKE (the only non-social category) may be responsible for such effects, we conducted two additional experiments. First, we observed similar effects as SNAKE using a categorically distinct, non-threatening FROG label among SELF and OTHER labels (Experiment 2A), suggesting that threat may not be responsible for SNAKE benefits. Second, we attempted to create a highly concrete and imageable, but not self-identified, social label (GREG) by linking the label to the experimenter. We find that this label can elicit performance benefits similar to SELF over OTHER. We conclude that self-relevance effects in the label-matching paradigm should be carefully parsed into effects due to selfidentification, and other factors such as concreteness.

\section{Experiment 1: Comparing self-identified with threatening labels}

We first sought to replicate self-identification effects (Experiment 1A), and then examine whether similar effects can be elicited via threatening associations (Experiments 1B and 1C). Our aim was to assess whether other forms of self-relevance are able to access the same salience-enhancing mechanisms that self-identification and self-affiliation seemingly access. Threatening stimuli are, by their very nature, self-relevant, but they are typically not identified with the self. There are many published examples of threatening stimuli capturing attention (Seeley et al., 2007; Most, Chun, Widders, \& Zald, 2005; Pessoa, 2009). We employ the label-matching paradigm while using two types of "threatening" labels in Experiments 1B and 1C. Establishing whether other types of associations can induce similar effects will more clearly define the boundaries of 'self-relevance' and the generality of the salience mechanism at play in prioritizing self-relevant information. This experiment incidentally addresses the possibility that demand characteristics may play a role in self-relevance effects, as the act of emphasizing the threatening nature of the labels may accentuate their status to the participant.

\section{Methods}

\section{Subjects}

Sixty-four subjects were recruited from the University of Delaware psychology subject pool: 20 subjects were recruited for Experiment 1A, 20 subjects were recruited for Experiment 1B, and 24 subjects were recruited for Experiment 1C.

\section{Apparatus}

The task was implemented using MATLAB (2015a Statistics Toolbox) and Psychtoolbox-3 (Brainard, 1997; Pelli, 1997; Kleiner, Brainard, \& Pelli, 2007) on a Linux PC. Participants were seated, unrestricted, approximately $52 \mathrm{~cm}$ away from a 17-in CRT computer monitor, and made responses using the keyboard.

\section{Stimuli}

The shape stimuli consisted of three distinct solid black shapes: circle, square, and triangle; the extent of each shape was approximately $5^{\circ} \times 5^{\circ}$. All word stimuli were presented in black uppercase font and varied from $5^{\circ} \times 1.6^{\circ}$ to $6.6^{\circ} \times 1.6^{\circ}$ depending on the number of letters in the label. During the experimental blocks a $0.5^{\circ} \times 0.5^{\circ}$ fixation cross was employed.

\section{Training procedure}

Subjects first completed a training block to learn to associate labels with shapes. In Experiment 1A, subjects were trained to associate the shape stimuli with standard self-relevance labels: SELF, FRIEND, and OTHER. In Experiment 1B, subjects learned associations corresponding to SELF, STRANGER, and OTHER. The subjects were instructed that the experiment was focusing on the effects of "stranger-danger," and that the stranger was "threatening," in order to boost the salience of the STRANGER label condition and enhance demand characteristics associated with a non-self-relevant label. In Experiment 1C, the labels that were assigned to the shape stimuli were: SELF, SNAKE, and OTHER. The participants were told that the SNAKE condition was representative of a "venomous snake." Independently for each participant, the three different shapes were randomly paired with the three labels. 
Subjects were informed of these associations, and then completed a short training task. On each trial of the training task one of the shapes appeared in the center of the screen, above three horizontally arrayed buttons labeled with each of the three terms, with label position randomized from trial to trial. Subjects were tasked with using the mouse to click the button that matched the shape's assigned label, and they were required to complete 30 consecutive correct trials before moving on to the experimental task. Participants were provided visual feedback on every trial in the form of the word 'Correct' for $1000 \mathrm{~ms}$ in green for correct trials and the word 'Incorrect' for $3000 \mathrm{~ms}$ in red for incorrect trials.

\section{Experimental procedure}

During the experimental task, participants responded to shape/ label pairs using the keyboard. On each trial, a fixation cross appeared for $500 \mathrm{~ms}$ in the middle of the screen, followed by a shape and a label. Either the shape or the label appeared above fixation, and the other below fixation, with position randomized from trial-to-trial. Participants responded by pressing the "M" key if the label matched the one assigned to the shape during training, and the "Z" key if they mismatched. Participants were instructed to respond as quickly as possible while maintaining as high an accuracy as possible. After the response, subjects were then presented with the same feedback stimuli as used in the training procedure ('Correct' for $500 \mathrm{~ms} /$ 'Incorrect' for $1000 \mathrm{~ms}$ ). The fixation cross designating the next trial appeared immediately after the feedback stimuli. Participants completed four blocks of the experimental task, consisting of 120 trials per block, totaling 480 trials. Stimulus presentation and congruency were pseudorandomized such that each combination of label and shape occurred an equal number of times in each block.

\section{Participant exclusion}

A priori exclusion criteria were established such that RTs outside the range of $250-3000 \mathrm{~ms}$ excluded. If more than $10 \%$ of a participant's trials were eliminated, the participant was completely removed from the analysis. This excluded three subjects in Experiment 1A, two subjects were eliminated from Experiment 1B, and two from Experiment 1C.

\section{Results: Experiment 1A}

During the initial training period, participants were required to correctly identify 30 shapes and respond with the appropriate label. The average number of training trials required was 32.76 with a standard deviation of 4.68 .

For all experiments, reaction times (RTs) and accuracy means and standard deviations are summarized in Table 1 based on label and match between label and shape. Based on prior work showing that differences in performance for labels are primarily associated with congruent matches between labels and shapes (Sui et al., 2012), and that matches are verified more quickly and accurately than mismatches, we quantified label-wise performance as the congruence benefit for RTsthe difference between the incongruent and congruent RTs for a given label. Congruence benefits were used to assess labelspecific benefits because they control for differences in processing times for each of the labels - incongruent RTs are presumably affected by the same characteristics that influence processing of the label in congruent trials. In this and all subsequent RT analyses we focused primarily upon the labelspecific congruence benefit to RT (incongruent RT - congruent RT) (Fig. 1). However, where we observed significant deviations across incongruent RTs, we describe the results in greater detail.

A one-way, three-factor ANOVA examined the differences between the congruency benefits for the three different label types. The main effect of label was significant $[\mathrm{F}(2,32)=$ 33.17, partial- $\eta^{2}=0.675$, and $\left.P<0.001\right]$. Pairwise comparisons determined that the SELF congruence benefit was larger than that for FRIEND [ $\mathrm{t}(16)=5.45, \mathrm{~d}=2.73$, and $P<0.001]$ and OTHER labels $[\mathrm{t}(16)=7.11, \mathrm{~d}=3.56$, and $P<0.001]$. FRIEND labels also received a congruency benefit significantly larger than the OTHER label $[\mathrm{t}=2.90, \mathrm{~d}=1.45$, and $P<0.01]$.

Accuracy was overall high, as expected, as there was no strict limit placed on RTs or stimulus duration, although fast and accurate performance was emphasized. In order to compare performance in terms of accuracy, we calculated A' scores for each label (we employed A' instead of d' due to near-ceiling accuracy), classifying correct label-match responses as hits and incorrect responses to label mismatches as false alarms. The main effect of label on A' was significant $\left[\mathrm{F}(2,32)=9.80\right.$, partial $-\eta^{2}=0.380$, and $\left.P<0.001\right]$. Pairwise comparisons revealed higher A' for SELF labels compared to FRIEND $[\mathrm{t}(16)=4.66, \mathrm{~d}=2.33$, and $P<0.001]$, and a significantly higher A' for SELF compared to OTHER [t(16) = $3.38, \mathrm{~d}=1.69$, and $P=0.004]$. FRIEND label A' was not significantly different from the OTHER label $[\mathrm{t}(16)=0.73$, $\mathrm{d}=0.37$, and $P=0.48]$.

The observed pattern of RTs could not be accounted for by a simple speed accuracy trade-off-faster RTs were generally associated with higher accuracy. The SELF labeled condition showed performance benefits in both RT and accuracy performance compared to the other conditions. While the FRIEND label condition showed a benefit to RT, the accuracy was not significantly different than the OTHER condition.

\section{Results: Experiment 1B}

During the initial training period participants were required to correctly identify 30 shapes and respond with the appropriate 
Table 1 Summary mean (SD) reaction times and accuracies for Experiments 1A, 1B, and 1C

\begin{tabular}{|c|c|c|c|c|c|}
\hline Condition & Congruent RT (ms) & Incongruent RT (ms) & Congruent accuracy (ms) & Incongruent accuracy (ms) & $\mathrm{A}^{\prime}$ \\
\hline \multicolumn{6}{|c|}{ Experiment 1A $(N=17)$} \\
\hline Self & $815(134)$ & $1048(195)$ & $98.4(1.8)$ & $88.75(3.6)$ & $0.99(0.01)$ \\
\hline Friend & 948 (169) & $1045(181)$ & $94.4(4.5)$ & $87.5(3.4)$ & $0.97(0.02)$ \\
\hline Other & $1022(200)$ & $1056(206)$ & $90.6(6.9)$ & $84.81(3.9)$ & $0.97(0.03)$ \\
\hline \multicolumn{6}{|c|}{ Experiment 1B $(N=18)$} \\
\hline Self & $854(112)$ & $1042(135)$ & $97.7(1.9)$ & $95.21(5.2)$ & $0.98(0.02)$ \\
\hline Stranger & $957(150)$ & $1032(132)$ & $96.1(4.8)$ & $94.51(4.7)$ & $0.98(0.03)$ \\
\hline Other & $971(151)$ & $1050(112)$ & $91.7(7.9)$ & $96.39(4.2)$ & $0.97(0.04)$ \\
\hline \multicolumn{6}{|c|}{ Experiment $1 \mathrm{C}(N=22)$} \\
\hline Self & $807(105)$ & 952 (119) & $98.0(1.7)$ & $96.2(3.2)$ & $0.99(0.01)$ \\
\hline Snake & 846 (129) & $980(132)$ & $95.3(5.4)$ & $95.9(3.4)$ & $0.98(0.02)$ \\
\hline Other & $892(113)$ & $950(123)$ & $94.1(4.9)$ & $96.5(3.9)$ & $0.98(0.02)$ \\
\hline
\end{tabular}

label. The average number of training trials required was 36.22 with a standard deviation of 10.69 .

We compared RTs across label (SELF/STRANGER/ OTHER), and whether label was congruent with shape according to training (Table 1). A one-way, three-factor ANOVA examined the differences between the congruency benefits for the three different label types. The main effect of label was significant $\left[\mathrm{F}(2,34)=7.14\right.$, partial $-\eta^{2}=0.300$, and $\left.P<0.003\right]$. Pairwise comparisons revealed that the SELF congruence benefit was larger than the STRANGER $[\mathrm{t}(17)=2.81, \mathrm{~d}=1.36$, and $P=0.012$ ] and OTHER label congruence benefit $[\mathrm{t}(17)=2.99, \mathrm{~d}=1.45$, and $P=0.008]$. STRANGER labels did not produce a significantly greater congruence benefit compared to the OTHER label $[\mathrm{t}=-0.15, \mathrm{~d}=-$ 0.072 , and $P=0.88]$.

The main effect of label on A' was significant $[\mathrm{F}(2,34)$ $=3.66$, partial $-\eta^{2}=0.177$, and $\left.P=0.036\right]$. Pairwise comparisons showed that SELF label A' was not significantly different than STRANGER label $\mathrm{A}^{\prime}[\mathrm{t}(17)=1.32, \mathrm{~d}=$ 0.64 , and $P=0.21$, but was greater than OTHER label $\mathrm{A}^{\prime}[\mathrm{t}(17)=2.74, \mathrm{~d}=1.33$, and $P=0.014]$. STRANGER label A' was not significantly different than OTHER label $\mathrm{A}^{\prime}[\mathrm{t}(17)=1.37, \mathrm{~d}=0.66$, and $P=0.19]$.
Accuracy was high and the results observed for RTs could not be accounted for by a simple speed accuracy trade-off. Consistent with RTs, SELF trials had the highest overall accuracy, but the benefit in accuracy was only significant compared with OTHER and not STRANGER.

\section{Results: Experiment 1C}

During the initial training period, participants were required to correctly identify 30 shapes and respond with the appropriate label. The average number of training trials required was 34.45 with a standard deviation of 10.53 .

We compared RTs across label (SELF/SNAKE/OTHER), and whether the label was congruent with shape according to training (Table 1). An ANOVA examined the differences between the congruency benefits for the three different label types. The main effect of label was significant $[\mathrm{F}(2,42)=$ 7.735, partial $-\eta^{2}=0.270$, and $\left.P=0.001\right]$. Pairwise comparisons determined that the SELF congruence benefit was not larger than the SNAKE $[\mathrm{t}(21)=0.58, \mathrm{~d}=0.25$, and $P=0.570]$, but was larger than OTHER labels $[\mathrm{t}(21)=3.59, \mathrm{~d}=1.57$, and $P=$ 0.002]. SNAKE labels congruence benefit was larger than that of the OTHER labels $[\mathrm{t}=2.78, \mathrm{~d}=1.21$, and $P=0.011]$.
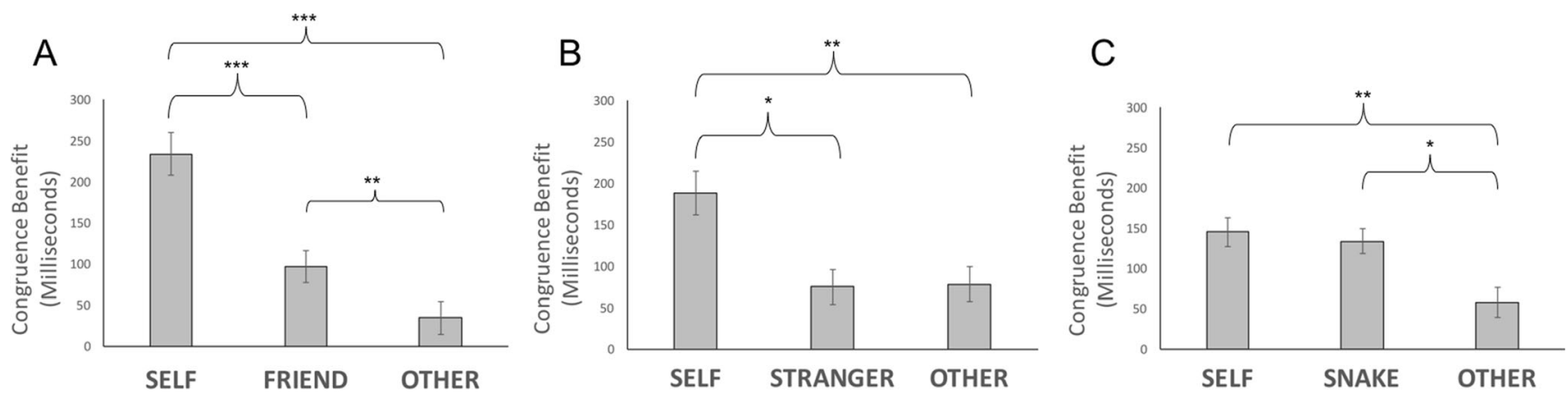

Fig. 1 a Congruence differences for the three label conditions in the Sui et al. (2012) replication. b Experiment 1B congruence benefits. c Experiment $1 \mathrm{C}$ congruence benefits. $(* P<.05 ; * * P<.01 ; * * * P<.001)$ 
The main effect of label on $A^{\prime}$ was significant $[F(2,42)=$ 4.31, partial $-\eta^{2}=0.170$, and $\left.P=0.020\right]$. Pairwise comparisons showed that SELF A' was significantly higher than SNAKE $\mathrm{A}^{\prime}[\mathrm{t}(21)=2.43, \mathrm{~d}=1.06$, and $P=0.024]$, and was also higher than the OTHER label $[\mathrm{t}(21)=2.40, \mathrm{~d}=1.05$, and $P=0.026]$. A' for SNAKE was not significantly different from the OTHER label's $[\mathrm{t}(21)=0.44, \mathrm{~d}=0.19$, and $P=0.66]$.

Accuracy was near ceiling for all conditions. Nevertheless, SELF responses were significantly more accurate than both SNAKE and OTHER, implying a benefit in terms of accuracy for SELF trials that was not observed in RTs, compared with SNAKE. SNAKE trial RT benefits over OTHER could not be explained by speed-accuracy tradeoff, given the statistical equivalence of accuracy across these conditions.

In Experiment 1C, there was a significant difference in the incongruent trial conditions. The incongruent value of the SNAKE label condition was significantly slower than SELF, $[\mathrm{t}(21)=-2.53, \mathrm{~d}=1.10, P=.020]$, as well as OTHER labels, $[\mathrm{t}(21)=-2.21, \mathrm{~d}=.964, \mathrm{p}=.038]$. Given this difference amongst the incongruent trials we conducted a post-hoc $2 \times 2$ ANOVA comparing the congruent and incongruent trials for the SELF and SNAKE label conditions. We found a significant main effect of label, $\left[\mathrm{F}(1,21)=8.53\right.$, partial- $\eta^{2}=.289, P=$ $.008]$, as well as a significant effect of congruence, $[\mathrm{F}(1,21)=$ 111.12, partial- $\left.\eta^{2}=.841, P<.001\right]$. However, the interaction between label and congruence was not significant, $[\mathrm{F}(1,21)=$ .333 , partial $\left.-\eta^{2}=.016, P=.57\right]$. Additionally, congruent reaction times for the SNAKE label were significantly faster than congruent OTHER label responses, $[\mathrm{t}(21)=-2.14, \mathrm{~d}=-.93, P$ $=.045]$. These results imply that the congruence benefit to SNAKE was reliable and no smaller than that to SELF, and that a benefit for SNAKE was observed over OTHER even when considering only congruent RTs.

\section{Discussion}

The results of Experiment $1 \mathrm{~A}$ replicated the principal findings of Sui et al. (2012), in that the congruence benefits were greater for the self-relevant stimulus, compared with FRIEND and other label conditions. FRIEND matches were verified faster than OTHER matches, suggesting that social affiliation with the concept represented by the FRIEND label is apparently adequate to speed responses compared to the most socially distant labeled concept, though not to the same degree as a self-identified stimulus.

In Experiment 1B there was no difference between performance in the STRANGER condition compared to the OTHER label. This pattern of results provides evidence for two claims. First, it is possible that benefits of self-relevant labels are produced by demand characteristics — participants may develop hypotheses concerning the aims of label-matching experiments that induce benefits for SELF labels, rather than accessing a particular top-down salience-enhancing mechanism. Our instructions in Experiment 1B were intentionally and particularly transparent in drawing attention to the STRANGER category and emphasizing its importance. Nevertheless, no comparable benefit was evident for STRANGER over OTHER labels, implying that the benefit of SELF is not likely due to experimental demand characteristics.

However, in Experiment 1C, the SNAKE label did provide a significant performance benefit compared with OTHER, and this benefit was not distinguishable from the benefit to SELF in terms of RT congruency effects. A small but reliable benefit in terms of accuracy was evident for SELF compared with SNAKE, however. The results of Experiment $1 \mathrm{C}$ demonstrate that RT benefits in the label-matching paradigm are not exclusive to self-identified or socially affiliated concepts.

We observed a difference between the incongruent conditions in Experiment $1 \mathrm{C}$, which we did not observe in any of the earlier experiments, such that SNAKE incongruent conditions yielded slower RTs compared with SELF and OTHER incongruent conditions. One possibility is that the SNAKE label alone may capture attention or evoke an emotional response, causing a delay not imposed upon SELF and OTHER labels. The presence of this difference in the incongruent conditions motivated a follow up ANOVA to see if there was an interaction of label and congruence in just the SELF and SNAKE conditions. No interaction was found, implying that the congruence benefits of SELF and SNAKE is of the same magnitude, with the SELF labels simply yielding faster RTs compared to SNAKE labels overall.

While in Experiment 1C, we observed a clear benefit in RT performance for the SNAKE compared with OTHER label, we did not observe a corresponding difference in accuracy. The SELF label was consistently the fastest, and most accurate condition. Although numerically small, the statistically significant benefit for SELF vs. SNAKE in terms of A' may represent true self-relevance effects, or be due to other factors that differentiate SELF and SNAKE.

We considered multiple possibilities concerning the benefit that was observed for SNAKE but not for STRANGER. First, the SNAKE condition may access a concept more naturalistically associated with threat, eliciting the action of the same or similar mechanisms as responsible for benefits of self-identified stimuli. Alternatively, SNAKE may be an "oddball" labelperhaps label-matching benefits accrue to conceptually distinct labels within the context of an experiment. In Experiments 1A and $1 \mathrm{~B}$, the non-OTHER, non-SELF category shares characteristics with the other labels, in that they are both social entities. In Experiment 1C, SNAKE is conceptually distinct - the only non-social/animal label involved. The fact that SELF retains priority over OTHER in Experiment 1C suggests that it is not this oddball status that explains SELF vs. OTHER performance benefits. Nevertheless, we address this possibility further in Experiment 2. 
Finally, it is possible that SNAKE is associated with congruency benefits because it is a specific, concrete, and/or imaginable term, whereas FRIEND, OTHER and STRANGER are relatively less specific, more abstract, and less imaginable. This possibility has implications for self-labels, which may also be more specific, concrete, and/or imageable than typical comparison labels. This might also explain why FRIEND congruence benefits are greater than OTHER. In the OTHER condition, it is possible that participants are less able (or less inclined) to effectively activate a specific representation of "some other person" from memory, hindering association, and thus performance in the matching task. In the FRIEND label condition, it is possible that participants are activating either one or many representations of friends, possibly causing the partial benefit observed by Sui and colleagues, moderated by the fact that the term is still relatively abstract, and matched to multiple social entities (compared with the singular self). This could also explain the differences we see in the two threatening conditions in Experiment $1 \mathrm{~B}$ and $1 \mathrm{C}$, where STRANGER is relatively abstract and may not activate a specific concrete concept, whereas SNAKE evokes a more concrete concept.

\section{Experiment 2: Oddball status and concreteness}

The goal of Experiment 2 was to investigate the differences between the threatening conditions in both Experiment $1 \mathrm{~B}$ and $1 \mathrm{C}$, and why there is an obvious benefit to performance in the "venomous snake" condition compared to the "dangerous stranger". First, in Experiment 2A, we eliminated the threatening component of the stimuli while maintaining the concrete nature of the SNAKE label, labeling the critical shape as a FROG (described as "a harmless frog" during instruction). If the effect in Experiment $1 \mathrm{C}$ is due to the threatening component of the stimuli, we expect to see no comparable benefit to performance towards the frog label (this choice was motivated by previous visual search research that found a benefit for searching for snakes compared with frogs, suggesting that frogs are not perceived as threats; LoBue \& DeLoache, 2008). In Experiment 2B, we replace the critical condition with a categorically social, but highly concrete label, GREG, the name of the researcher administering the study. This addresses the possibility that the difference in congruency benefits between the STRANGER and SNAKE conditions may be a result of the difference in the concreteness or imageability of the labels themselves, with the "venomous snake" label being more imageable compared to the ambiguously described "dangerous stranger".

\section{Methods}

Methods for Experiment 2 were identical to those of Experiment 1 with these following changes.
Subjects

Forty-three subjects new were recruited from the same subject pool; 21 subjects were recruited for Experiment $2 \mathrm{~A}$, and 22 subjects were recruited for Experiment $2 \mathrm{~B}$.

\section{Training procedure}

Subjects in Experiment 2 underwent the same training procedure as in Experiment 1 with different sets of labels. Participants in Experiment 2A were trained on the labels: SELF, FROG, and OTHER. Participants in Experiment 2B were trained on the labels: SELF, GREG, and OTHER. When participants in experiment $2 \mathrm{~B}$ were presented with the GREG shape/label pair during initial label assignment, they were simultaneously presented with a picture of the researcher running the study to enhance imageability and concreteness of the GREG label. The image did not appear during training trials.

\section{Participant exclusion}

The same a priori criteria were applied as in Experiment 1. This resulted in exclusion of three subjects in Experiment 2B.

\section{Results: Experiment 2A}

During the initial training period participants were required to correctly identify 30 shapes and respond with the appropriate label. The average number of training trials required was 43.10 with a standard deviation of 20.80 .

A one-way three factor ANOVA was conducted on incongruent reaction times, which were not statistically different from one another $\left[\mathrm{F}(2,40)=0.547, P=0.58\right.$ and partial $-\eta^{2}=$ $0.027]$. Thus, we relied primarily upon the congruence benefit in our analysis.

We compared congruence benefits across labels (SELF/FROG/OTHER). A one-way, three-factor ANOVA revealed that the main effect of label was significant $[\mathrm{F}(2,40)=$ 9.75, partial $-\eta^{2}=0.328$, and $\left.P<0.001\right]$. Pairwise comparisons showed that the SELF congruence benefit was not different than the FROG label congruence benefit $[\mathrm{t}(20)=1.65, \mathrm{~d}=0.433$, and $P=0.115]$. SELF label congruency benefit was significantly larger than OTHER label congruence benefit $[\mathrm{t}(20)=4.06, \mathrm{~d}=$ 1.26 , and $P=0.001]$. FROG labels produced a significantly greater congruence benefit compared to the OTHER label as well $[\mathrm{t}(20)=2.98, \mathrm{~d}=0.750$, and $P=0.007]$. Congruent reaction times for SELF stimuli were marginally faster than congruent FROG reaction times $[\mathrm{t}(20)=-2.054, \mathrm{~d}=-0.92, P=0.053]$.

Accuracy was overall high, as expected, and the results observed for response times could not be accounted for by a simple speed accuracy trade-off. The main effect of label on A' was trending, but not significant $\left[\mathrm{F}(2,40)=2.57\right.$, partial- $\eta^{2}$ $=0.114$, and $P=0.089]$. Pairwise comparisons showed that 
SELF label A' was significantly higher than FROG label A' $[\mathrm{t}(20)=2.42, \mathrm{~d}=0.35$, and $P=0.025]$, and was also marginally greater than OTHER label $\mathrm{A}^{\prime}[\mathrm{t}(20)=2.01, \mathrm{~d}=0.48$, and $P=0.058]$. FROG label A' was not significantly different than OTHER label A' $[\mathrm{t}(20)=0.56, \mathrm{~d}=0.14$, and $P=0.58]$.

\section{Results: Experiment 2B}

During the initial training period participants were required to correctly identify 30 shapes and respond with the appropriate label. The average number of training trials required was 42.58 with a standard deviation of 16.63 .

A one-way three factor ANOVA was conducted to confirm that incongruent reaction times were not statistically different from one another $\left[\mathrm{F}(2,36)=0.497, \mathrm{p}=0.61\right.$ and partial $-\eta^{2}=$ $0.027]$. We thus relied on the congruence benefit calculation to analyze RTs.

We compared congruence benefits across labels (SELF/GREG/OTHER; Table 2 and Fig. 2b). A one-way, three-factor ANOVA examined the differences between the congruency benefits for the three different label types. The main effect of label was significant $\left[\mathrm{F}(2,36)=8.32\right.$, partial $-\eta^{2}=$ 0.316 , and $p=0.001]$. Pairwise comparisons revealed that the SELF congruence was no different than GREG $[\mathrm{t}(18)=0.976, \mathrm{~d}$ $=0.317$, and $P=0.34]$; SELF congruence benefit was significantly larger than OTHER label congruence benefit $[\mathrm{t}(18)=3.36$, $\mathrm{d}=1.27$, and $P=0.003$ ]; and GREG labels produced a significantly greater congruence benefit compared to the OTHER label, as well $[\mathrm{t}(18)=3.09, \mathrm{~d}=1.10$, and $\mathrm{p}=0.006]$. Congruent reaction time for SELF stimuli were faster than congruent GREG reaction times $[\mathrm{t}(18)=-2.30, \mathrm{~d}=-1.02, P=0.033]$.

Accuracy was overall high, as expected, and effects were consistent with response times, such that results observed for response times could not be accounted for by a simple speed accuracy trade-off. The main effect of label on $A^{\prime}$ was significant $\left[\mathrm{F}(2,36)=6.46\right.$, partial $-\eta^{2}=0.264$, and $P=$ 0.004]. Pairwise comparisons showed that SELF label A' was not significantly different than GREG label A' $[\mathrm{t}(18)=0.42, \mathrm{~d}=$
0.12 , and $P=0.68]$, but was greater than OTHER label $A^{\prime}$ $[\mathrm{t}(18)=2.59, \mathrm{~d}=0.74$, and $P=0.018]$. GREG label $\mathrm{A}^{\prime}$ was also significantly higher than OTHER label $\mathrm{A}^{\prime}[\mathrm{t}(18)=3.12, \mathrm{~d}=$ 0.61 , and $P=0.006]$.

\section{Discussion}

In both Experiment 2A and 2B, the critical labels, FROG and GREG, showed a significant and similar congruence benefit as the self-relevant shape/label pair. Both FROG and GREG RT congruence benefits were greater than the baseline condition of OTHER, and were not statistically different from the SELF-labeled stimuli.

Accuracies for all experiments were overall high. A numerically small but statistically significant difference in accuracy (as reflected in A') was observed in Experiment 2A, such that SELF label decisions were more accurate than FROG label decisions, just as they were for SNAKE in Experiment $1 \mathrm{C}$. This suggests the possibility of a residual self-relevance effect observable only in accuracy, given that the SELF labels were consistently associated with the highest accuracies. This possibility cannot be eliminated. However, SELF accuracy was indistinguishable from GREG accuracy, supporting the possibility that other factors may have played a role in SELF vs. FROG and SELF vs. SNAKE differences in accuracy.

The results of Experiment 2A suggests that the benefits observed for SNAKE labels over OTHER labels may not have been the result of threat. The remaining possibilities considered were the oddball status and concrete nature of the SNAKE and FROG labels. In Experiment 2B we constructed a non-oddball, non-self-relevant social condition with high concreteness and imageability - the subject interacted with and was shown a picture of the referent of GREG prior to training to enhance concreteness and imageability. GREG performance was both highly accurate and resulted in comparable congruent benefits as SELF labels.

Table 2 Summary mean (SD) reaction times and accuracies for Experiments 2A and 2B

\begin{tabular}{|c|c|c|c|c|c|}
\hline Condition & Congruent RT (ms) & Incongruent RT (ms) & Congruent accuracy (ms) & Incongruent accuracy (ms) & $\mathrm{A}^{\prime}$ \\
\hline \multicolumn{6}{|c|}{ Experiment 2A $(N=21)$} \\
\hline Self & $837(135)$ & $1001(180)$ & $97.3(3.3)$ & $97.5(2.0)$ & $.99(.01)$ \\
\hline Frog & $886(158)$ & $1010(1778)$ & $96.6(24.59)$ & $96.4(5.0)$ & $.98(.01)$ \\
\hline Other & $936(182)$ & $993(189)$ & $94.6(135)$ & $97.8(2.4)$ & $.98(.02)$ \\
\hline \multicolumn{6}{|c|}{ Experiment $2 \mathrm{~B}(N=19)$} \\
\hline Self & $894(127)$ & $1060(180)$ & $97.6(1.6)$ & $97.2(2.1)$ & $.99(.01)$ \\
\hline Greg & $937(142)$ & $1076(147)$ & $97.7(1.6)$ & $97.0(3.2)$ & $.98(.01)$ \\
\hline Other & $1023(181)$ & $1060(157)$ & $94.3(6.2)$ & $96.6(2.9)$ & $.98(.02)$ \\
\hline
\end{tabular}



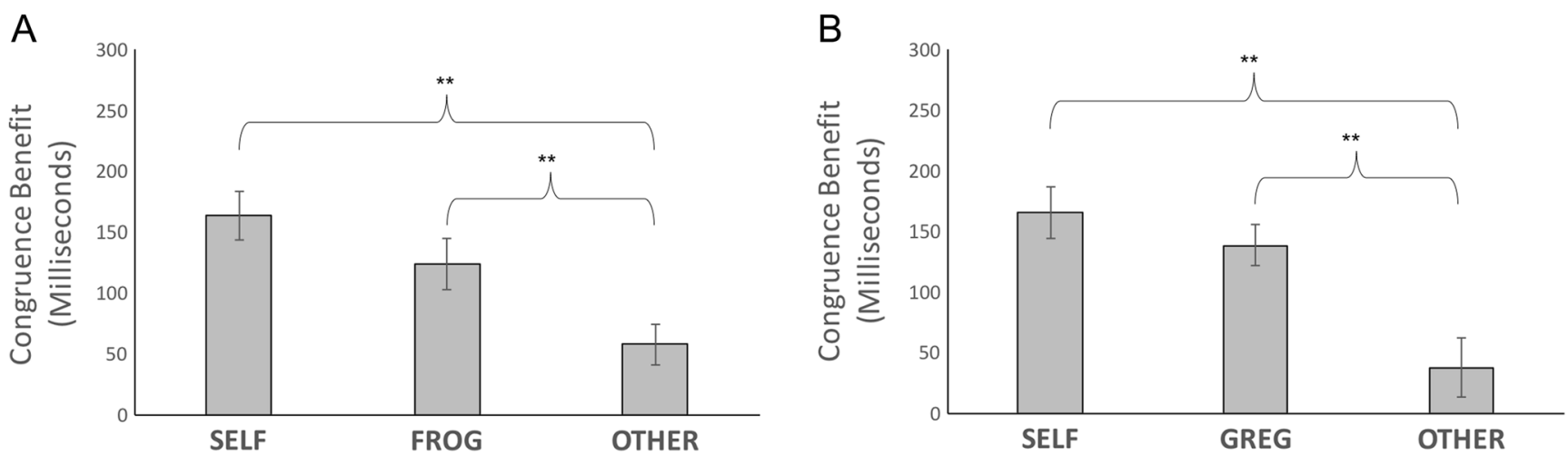

Fig. 2 a Congruence differences for the three label conditions in Experiment 2 A. b Experiment $2 \mathrm{~B}$ congruence benefits. $(* P<.05$; $* * P<.01 ; * * * P<.001)$

\section{General discussion}

We produced effects resembling those of putative selfrelevance effects using non-self-identified labels. In Experiment 1A, we replicated the basic self-relevance effect (with SELF, FRIEND, and OTHER labels), demonstrating that it is a robust phenomenon. Subsequent experiments replaced the FRIEND label with novel labels to compare effects of the new label with a control (OTHER) and the selfidentified label known to produce large congruency benefits (SELF). In Experiment 1B, we used STRANGER, a secondary label sometimes used as a control, but we additionally introduced demand characteristics and a threatening description. Nevertheless, no comparable congruence benefit was observed for the STRANGER label, suggesting that selfrelevance effects are not due to demand characteristics and may not be affected by threat. However, in Experiment 1C we introduced SNAKE, which is naturalistically associated with threat and subject to a lifetime of experience that associates this concept with threat. This label appeared to receive similar congruency benefits as the SELF label. Since we did not observe the effect of threat in Experiment 1B, we sought to control for different potentially confounding factors, such as concreteness of the concept evoked by the label and categorical differences. In Experiment 2A, we eliminated the possibility that threat was necessary to produce a similar benefit. The FROG label elicited a significant RT congruence benefit in the task, comparable to the SELF label. In Experiment 2B, we introduced the social GREG label, and associated it with the identity and image of the researcher running participants in the study. The GREG label also showed a congruence and accuracy benefit similar to the SELF label.

Throughout the experiments, SELF performance was always slightly better than other labels. It is worth noting, however, that in the case of GREG the effects were nearly equivalent, although the slightly (but significantly) faster performance in congruent conditions leaves the door open for selfrelevance benefits. We do not wish to (and cannot support the) claim that we have overturned the possibility that selfrelevance contributes to the label-matching results. However, we do claim that it is likely that the effect of self-relevance is, at a minimum, grossly over-estimated in the absence of control labels that have high specificity. The remaining performance edge for self-relevant labels could be true selfrelevance effects, or due to some other factor, such as lifelong experience flexibly associating the self with arbitrary stimuli in the world.

Compelling evidence for a unique self-relevance benefit in label-matching comes from a neuroimaging study. Sui, Rotshtein, and Humphreys (2013) examined fMRI activation while subjects judged shape-label pairs (self, friend, and stranger). Self-match pairs elicited stronger activation than stranger-match pairs only in left lateral posterior temporal sulcus (LpSTS) and ventromedial prefrontal cortex (vmPFC), two regions previously associated with self-referential thinking. Increased activity in these regions was also linked to greater self-relevance benefits. However, vmPFC is associated with many other functions as well, including associative processing (e.g., Behrens, Hunt, Woolrich, \& Rushworth, 2008), while both vmPFC and pSTS are linked with a putative "ventral attentional network" that is related to more automatic attentional orienting (Corbetta \& Shulman, 2002; Vossel, Geng, \& Fink, 2014). Stranger $>$ self yielded a much broader set of regions, many of them part of the dorsal frontoparietal control network associated with effortful processing (Corbetta \& Shulman, 2002).

Humphreys and Sui (2016) propose that vmPFC/LpSTS form a critical component of a "self-attention network" that interfaces with other attentional mechanisms. An alternative possibility, suggested by our data, is that associative memory mechanisms are more proficient for concrete compared with abstract concepts. A stronger associative link may elicit the function of the more automatic ventral attention network when doing the label-matching task, while weaker associative memory may elicit greater involvement of the dorsal attentional network. 
Stepping aside from the issue of self-relevance, our study suggests that concrete words may form more powerful, arbitrary associative links to arbitrary non-word stimuli. While previous work has shown multiple benefits of concreteness on performance, such as on recognition memory (Paivio, 1986; Tse \& Altarriba, 2007; Paivio, et al., 1966) and lexical decisions (Bleasdale, 1987; de Groot, 1989; Whaley, 1978; Lagishetti \& Goswami, 2012), to our knowledge, the effects of word concreteness on relational memory to arbitrary non-word stimuli suggested here are novel. However, the finding is highly consistent with the fact that mental imagery improves associative learning (Bower, 1970), and that associations between concrete word pairs are better acquired than between abstract word pairs (Paivio, 1965). Speculatively, the result may be related to imagery strategies, e.g., picturing a frog resting on a circular lily pad to remember the frog-circle association. The same idea may help explain putative self-relevance effects, as one might, for example, imaging holding and possessing a triangle in one's hand to remember that association. This possibility deserves future attention.

A principal limitation of the current study is the simple nature of our demonstration that similar effects to self-relevance may be induced without identification of the self with the target label. Experiments 2A and 2B, in particular, provide an important proof-of-concept example that variation in non-self-properties results in strong variations in label-matching performance. This leaves open the possibility that the evoked benefits depend on separate mechanisms compared with the observed benefits of self-relevant labels.

In all experiments, the mean value of the congruent selfrelevant stimulus reaction time response was the fastest, and the self-labeled responses were most accurate $\left(A^{\prime}\right)$, suggesting that there is still evidence for a unique self-relevant benefit to performance; yet, the benefits to self-relevant stimuli are clearly greatly overestimated in the absence of distinctive concrete label comparison conditions. The source of the additional benefit may reflect true self-relevance effects via the engagement of a selfspecific attentional network, or may be due to other factors, such as lifetime experience associating arbitrary stimuli with the self, compared with SNAKE, FROG, GREG, and OTHER.

Our results highlight the need to critically examine alternative hypotheses to self-relevance prior to accepting the existence of a complex dedicated mechanism to self-relevance, per se. Future studies should seek to more closely match selected labels on dimensions that are correlated with the degree of self-relevance.

Acknowledgements This research was supported by NSF BCS 1558535 and NSF OIA 1632849.

\section{References}

Behrens, T. E. J., Hunt, L. T., Woolrich, M. W., \& Rushworth, M. F. S. (2008). Associative learning of social value. Nature, 456, 245-250.
Binder, J. R., Westbury, C. F., McKiernan, K. A., Possing, E. T., \& Medler, D. A. (2005). Distinct brain systems for processing concrete and abstract concepts. Journal of Cognitive Neuroscience, 17(6), 905-917.

Bleasdale, F. A. (1987). Concreteness-dependent associative priming: Separate lexical organization for concrete and abstract words. Journal of Experimental Psychology: Learning Memory and Cognition, 13(4), 582-594.

Bower, G. H. (1970). Imagery as a relational organizer in associative learning 1. Journal of Verbal Learning and Verbal Behavior, 533, 529-533.

Brainard, D. H. (1997). The psychophysics toolbox. Spatial Vision, 10, 433-436.

Cisler, J. M., Bacon, A. K., \& Williams, N. L. (2009). Phenomenological characteristics of attentional biases towards threat: A critical review. Cognitive Therapy and Research, 33, 221-234.

Corbetta, M., \& Shulman, G. L. (2002). Control of goal-directed and stimulus-driven attention in the brain. Nature Reviews. Neuroscience, 3, 201-215.

de Groot, A. M. B. (1989). Representational aspects of word imageability and word frequency as assessed through word association. Journal of Experimental Psychology: Learning, Memory, and Cognition, 15, 824-845.

Humphreys, G. W., \& Sui, J. (2016). Attentional control and the self: The self attention network (SAN). Cogn Neurosci, 7, 5-29.

Johnson, S. C., Baxter, L. C., Wilder, L. S., Pipe, J. G., Heiserman, J. E., \& Prigatano, G. P. (2002). Neural correlates of self-reflection. Brain: A Journal of Neurology, 125(8), 1808-1814.

Keenan, J. P., Wheeler, M. A., Gallup, G. G., \& Pascual-Leone, A. (2000). Self-recognition and the right prefrontal cortex. Trends in Cognitive Sciences, 4(9), 338-344.

Kelley, W. M., Macrae, C. N., Wyland, C. L., Caglar, S., Inati, S., \& Heatherton, T. F. (2002). Finding the self? an event-related fMRI study. Journal of Cognitive Neuroscience, 14(5), 785-794.

Kiehl, K. A., Liddle, P. F., Smith, A. M., Mendrek, A., Forster, B. B., \& Hare, R. D. (1999). Neural pathways involved in the processing of concrete and abstract words. Human Brain Mapping, 233(7), 225-233.

Kircher, T. T. J., Senior, C., Phillips, M. L., Benson, P. J., Bullmore, E. T., Brammer, M., ... David, A. S. (2000). Towards a functional neuroanatomy of self-processing: Effects of faces and words. Cognitive Brain Research, 10, 133-144.

Kleiner, M., Brainard, D., Pelli, D, (2007). What's new in psychtoolbox-3? Perception, 36, ECVP Abstract Supplement.

Lagishetti, S. K., \& Goswami, S. P. (2012). Measurement of reaction time for processing of concrete and abstract words. Journal of All India Institute of Speech and Hearing JAIISH, 31, 139-144.

LoBue, V., \& DeLoache, J. S. (2008). Detecting the snake in the grass. Psychological Science, 19(3), 284-289.

Macrae, C. N., Moran, J. M., Heatherton, T. F., Banfield, J. F., \& Kelley, W. M. (2004). Medial prefrontal activity predicts memory for self. Cerebral Cortex, 14(6), 647-654.

MATLAB and Statistics Toolbox Release (2015a). The MathWorks, Inc.: Natick, MA.

Most, S. B., Chun, M. M., Widders, D. M., \& Zald, D. H. (2005). Attentional rubbernecking: Cognitive control and personality in emotion-induced blindness. Psychonomic Bulletin \& Review, 12(4), 654-661.

Notebaert, L., Crombez, G., Van Damme, S., De Houwer, J., \& Theeuwes, J. (2011). Signals of threat do not capture, but prioritize, attention: A conditioning approach. Emotion, 11(1), 8189.

O’Doherty, J., Winston, J., Critchley, H., Perrett, D., Burt, D. M., \& Dolan, R. J. (2003). Beauty in a smile: The role of medial orbitofrontal cortex in facial attractiveness. Neuropsychologia, 41(2), 147-155. 
Ohman, A., Flykt, A., \& Esteves, F. (2001). Emotion drives attention: Detecting the snake in the grass. Journal of Experimental Psychology: General, 130(3), 466-478.

Paivio, A. (1965). Abstractness, imagery, and meaningfulness in pairedassociate learning. Journal of Verbal Learning and Verbal Behavior, 4, 32-38.

Paivio, A. (1986). Mental representations. New York: Oxford University Press.

Paivio, A., Yuille, J. C., \& Madigan, S. A. (1968). Concreteness, imagery, and meaningfulness values for 925 nouns. Journal of Experimental Psychology Monograph Supplement, 76(1), 1-25.

Paivio, A., Yuille, J. C., \& Smythe, P. C. (1966). Stimulus and response abstractness, imagery, and meaningfulness and reported mediators in paired-associate learning. Canadian Journal of Psychology, 20, 362-378.

Pelli, D. G. (1997). The VideoToolbox software for visual psychophysics: Transforming numbers into movies. Spatial Vision, 10, 437-442.

Pessoa, L. (2009). How do emotion and motivation direct executive control? Trends in Cognitive Sciences, 13(4), 160-166.

Seeley, W. W., Menon, V., Schatzberg, A. F., Keller, J., Glover, G. H., Kenna, H., ... Greicius, M. D. (2007). Dissociable intrinsic connectivity networks for salience processing and executive control. 27(9):2349-2356.

Smith, S. D., Most, S. B., Newsome, L. A., \& Zald, D. H. (2006). An emotion-induced attentional blink elicited by aversively conditioned stimuli. Emotion, 6(3), 523-527.

Sui, J., He, X., \& Humphreys, G. W. (2012). Perceptual effects of social salience: Evidence from self-prioritization effects on perceptual matching. Journal of Experimental Psychology Human Perception and Performance, 38(5), 1105-1117.

Sui, J., \& Humphreys, G. W. (2015a). The integrative self: How selfreference integrates perception and memory. Trends in Cognitive Sciences, 19(12), 719-728.

Sui, J., \& Humphreys, G. W. (2015b). The interaction between self-bias and reward: Evidence for common and distinct processes. Quarterly Journal of Experimental Psychology, 68, 1952-1964.
Sui, J., \& Liu, C. H. (2009). Can beauty be ignored? Effects of facial attractiveness on covert attention. Psychonomic Bulletin \& Review, 16(2), 276-281.

Sui, J., Liu, M., Mevorach, C., \& Humphreys, G. W. (2015). The salient self: The left intraparietal sulcus responds to social as well as perceptual-salience after self-association. Cerebral Cortex, 25, $1060-1068$.

Sui, J., Liu, C. H., Wang, L., \& Han, S. (2009). Attentional orientation induced by temporarily established self-referential cues. Quarterly Journal of Experimental Psychology, 62, 844-849.

Sui, J., Rotshtein, P., \& Humphreys, G. W. (2013). Coupling social attention to the self forms a network for personal significance. Proceedings of the National Academy of Sciences of the Unites States of America, 110(19), 7607-7612.

Symons, C. S., \& Johnson, B. T. (1997). The self-reference effect in memory: A meta-analysis. Psychological Bulletin, 121(3), 371-394.

Tacikowski, P., Brechmann, a, Marchewka, a, Jednoróg, K., Dobrowolny, M., \& Nowicka, a. (2011). Is it about the self or the significance? An fMRI study of self-name recognition. Social Neuroscience, 6(1), 98-107. doi:10.1080/17470919.2010.490665.

Tse, C., \& Altarriba, J. (2007). Testing the associative-link hypothesis in immediate serial recall: Evidence from word frequency and word imageability effects. Memory, 6, 675-690.

Turk, D. J., Heatherton, T. F., Kelley, W. M., Funnell, M. G., Gazzaniga, M. S., \& Macrae, C. N. (2002). Mike or me? Self-recognition in a split-brain patient. Nature Neuroscience, 5(9), 841-842.R

Vossel, S., Geng, J. J., \& Fink, G. R. (2014). Dorsal and ventral attention systems: Distinct neural circuits but collaborative roles. The Neuroscientist, 20(2), 150-159.

Whaley, C. P. (1978). Word-nonword classification time. Journal of Verbal Learning and Verbal Behavior, 17, 143-154. 